\title{
A escolha do tempo em um modelo cosmológico quântico com cinco fluidos
}

\author{
Monerat, G. A. ${ }^{1 *}$; Alvarenga, F. G. ${ }^{2 \dagger}$; Silva, E. V. C. ${ }^{3+} ;$ Neto, G. de O. ${ }^{41}$ Gonçalves, S. V. \\ de B. $^{5 i}$; Fracalossi, . $^{6 \mathrm{t}}$. \\ ${ }^{1}$ Departamento de Modelagem Computacional, Universidade do Estado do Rio de Janeiro, Nova Friburgo, RJ, Brasil. \\ ${ }^{2}$ Departamento de Ciências Naturais, Universidade Federal do Espírito Santo, Vitória, ES, Brasil. \\ ${ }^{3}$ Departamento de Matemática, Física e Computação, Universidade do Estado do Rio de Janeiro, Resende, RJ, Brasil. \\ ${ }^{4}$ Departamento de Física, Universidade Federal de Juiz de Fora, Juiz de Fora, MG, Brasil. \\ ${ }^{5,6}$ Departamento de Física, Universidade Federal do Espírito Santo, Vitória, ES, Brasil. \\ * e-mail: germano.monerat@pq.cnpq.br; ${ }^{\dagger}$ e-mail: f.g.alvarenga@gmail.com; t e-mail: evasquez@uerj.br; 」 e-mail: \\ gilneto@fisica.ufjf.br; i e-mail: sergio.vitorino@pq.cnpq.br; t e-mail: rfracalossi@gmail.com
}

\begin{abstract}
Resumo
Neste trabalho propõe-se a quantização de um modelo cosmológico descrevendo o Universo primordial preenchido com cinco fluidos barotrópicos: radiação, poeira, vácuo, cordas cósmicas e paredes de domínio. Pretende-se identificar qual fluido é mais adequado para fornecer fenomenologicamente a variável temporal em acordo com a evolução do Universo observável. Através do emprego do método espectral de Galerkin, as soluções cosmológicas são obtidas e então comparadas.
\end{abstract}

\begin{abstract}
In this work we propose the quantization of a cosmological model describing the primordial Universe filled with five barotropic fluids: radiation, dust, vacuum, cosmic strings and domain walls. It is intended to identify which fluid is most appropriate to provide phenomenologically the temporal variable in accordance with the evolution of the observable Universe. By employing spectral Galerkin method, cosmological solutions are obtained and then compared.
\end{abstract}

Keywords (Palavras chaves): Cosmologia Quântica, Muitos fluidos, Problema do tempo.

\section{Introdução}

O fato de não haver uma teoria completa de gravitação quântica implica na necessidade de se testar os efeitos quânticos em diferentes regimes e modelos de Universo. Neste sentido, modelos cosmológicos quânticos [1] constituem exemplos simples nos quais as ideias de fenômenos gravitacionais quânticos podem ser testadas. $O$ cenário é 0 de minisuperespaço, no qual congela-se um número infinito de graus de liberdade e quantiza-se os remanescentes. A não explícita presença de uma variável do tipo tempo [2] pode ser contornada pela introdução fenomenológica de variáveis dinâmicas associadas a diferentes conteúdos materiais do Universo, com a pressão sendo expressa em termos de potenciais escalares de velocidades, dos quais um deles pode exercer o papel do tempo [3]. Deste modo, uma equação do tipo Schrödinger é estabelecida, o que possibilita obter a chamada função de onda do Universo e valores esperados do fator de escala do Universo. Neste trabalho quantiza-se um modelo de Universo preenchido com cinco fluidos barotrópicos: radiação, poeira, vácuo, cordas cósmicas e paredes de domínio, objetivando identificar qual fluido é mais adequado para fornecer a variável temporal em acordo com a evolução cosmológica do Universo.

\section{O Modelo}

Os modelos homogêneos de Friedmann-RobertsonWalker (FRW) com curvatura espacial k preenchidos com um dado fluido barotrópico $(p=\alpha \rho)$ podem ser representados pela Hamiltoniana [4]

$$
H=-\frac{p_{a}^{2}}{24 a}-6 k a+\frac{p_{T}}{a^{3 \alpha}},
$$

onde, $p_{a}$ e $p_{T}$ são respectivamente, os momentos canonicamente conjugados às variáveis fator de escala do Universo a e grau de liberdade do fluido perfeito $T$. Para um modelo com cinco fluidos, radiação $(\alpha=1 / 3)$, poeira $(\alpha=0)$, cordas cósmicas $(\alpha=-1 / 3)$, paredes de domínio $(\alpha=-2 / 3)$ e vácuo $(\alpha=-1)$, a Hamiltoniana pode 


\section{Encontro Científico de Física Aplicada}

ser estendida de modo a inserir as contribuições de todos os fluidos:

$$
\begin{aligned}
& H=p_{a}^{2}+144 k a^{2}-24 p_{\text {Trad }}-24 p_{\text {Tpoeira }} a- \\
& 24 p_{\text {Tcordas }} a^{2}-24 p_{\text {Tparedes }} a^{3}-24 p_{\text {Tvácuo }} a^{4} .
\end{aligned}
$$

Pode-se implementar agora o procedimento de quantização canônica em minisuperespaço e obter a correspondente equação de Wheeler-DeWitt

$$
\widehat{H} \Psi=0 .
$$

Substituindo os momentos por operadores correspondentes

$$
\hat{p}_{a}=-i \frac{\partial}{\partial a} \quad \text { e } \hat{p}_{T}=-i \frac{\partial}{\partial T},
$$

transforma-se a equação de Wheeler-DeWitt em uma genuína equação tipo-Schrödinger como consequência da presença linear dos momentos associados à variável $T$. Portanto, é possível introduzir o tempo fenomenologicamente de cinco maneiras diferentes, cada uma associada a um dado fluido.

No caso do fluido de cordas cósmicas, por exemplo, a escolha da introdução do tempo via $p_{\text {Tcordas }}$,estabelece a equação

$$
-\frac{\partial^{2} \Psi(\mathrm{a}, \mathrm{t})}{\partial a^{2}}+Q_{e f}(a) \Psi(a, t)=24 i \frac{\partial \Psi(a, T)}{\partial t}
$$

para a função de onda do Universo, onde o potencial efetivo é dado por:

$$
\begin{gathered}
Q_{e f}(a)=144 k a^{2}-24 p_{\text {Tradiação }}-24 p_{\text {Tpoeira }} a- \\
24 p_{\text {Tparedes }} a^{3}-24 p_{\text {Tvácuo }} a^{4}
\end{gathered}
$$

Acima o parâmetro temporal foi reescalonado $(T \rightarrow-t)$.

O valor esperado do fator de escala do Universo, assim como a densidade de probabilidades inicial, são obtidos a partir do método espectral de Galerkin [5]:

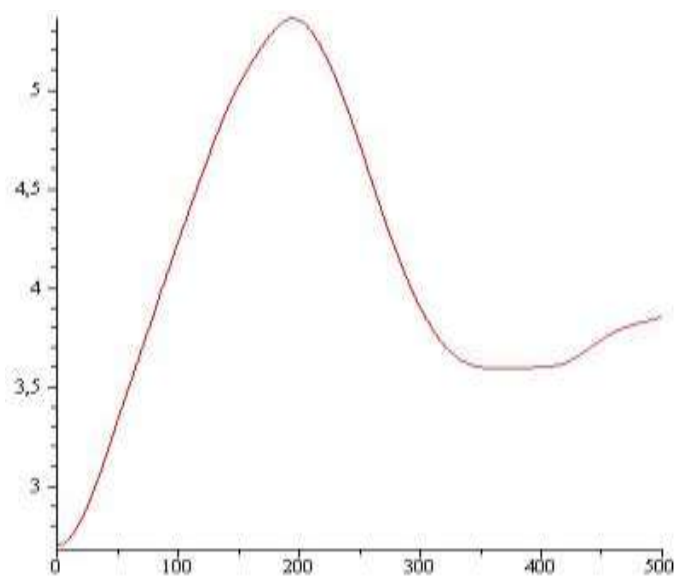

Figura 1: Valor esperado <a>, $\mathrm{k}=1$, em função do tempo $\mathrm{t}$ (cordas).

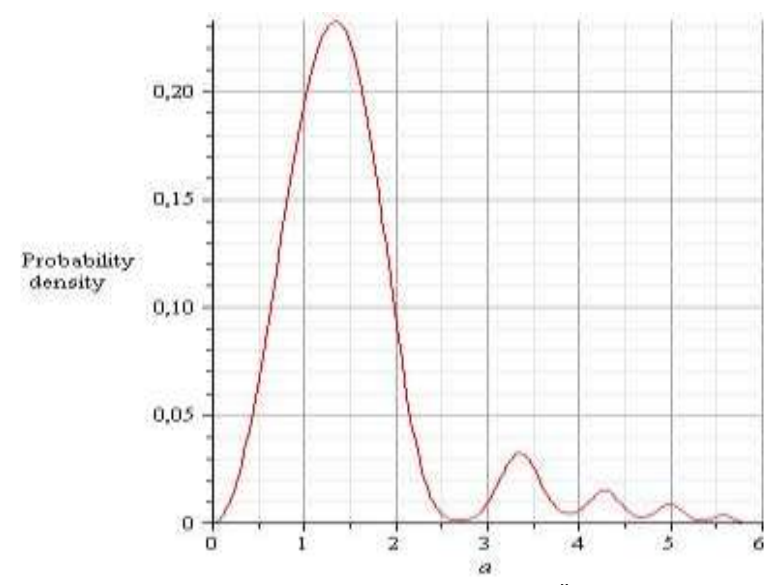

Figura 2: Densidade de probabilidade inicial $|\psi(a, 0)|^{2}$ (cordas).

No caso da escolha do vácuo para introdução do tempo, o potencial efetivo assume agora a forma

$$
\begin{gathered}
Q_{e f}(a)=144 k a^{2}-24 p_{\text {Tradiação }}-24 p_{\text {Tpoeira }} a- \\
24 p_{\text {Tcordas }} a^{2}-24 p_{\text {Tparedes }} a^{3} .
\end{gathered}
$$

O valor esperado e a densidade de probabilidade para o pacote de onda inicial são apresentados abaixo:

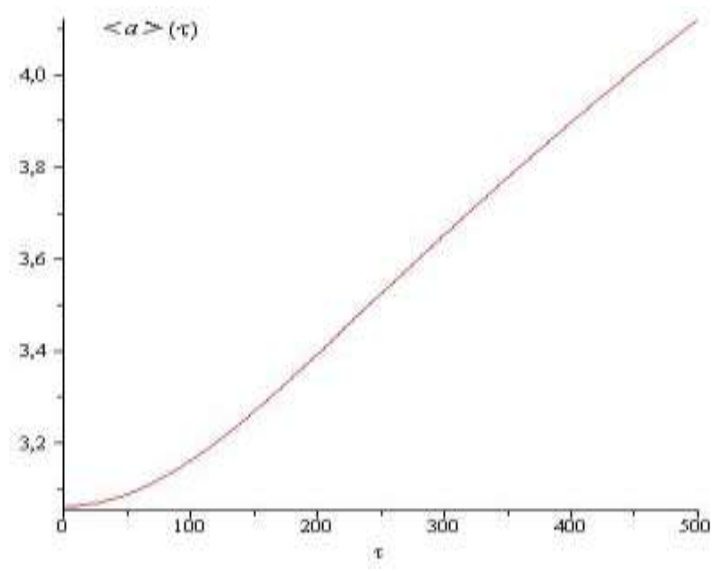

Figura 3: Valor esperado <a>, k=1, em função do tempo t (vácuo).

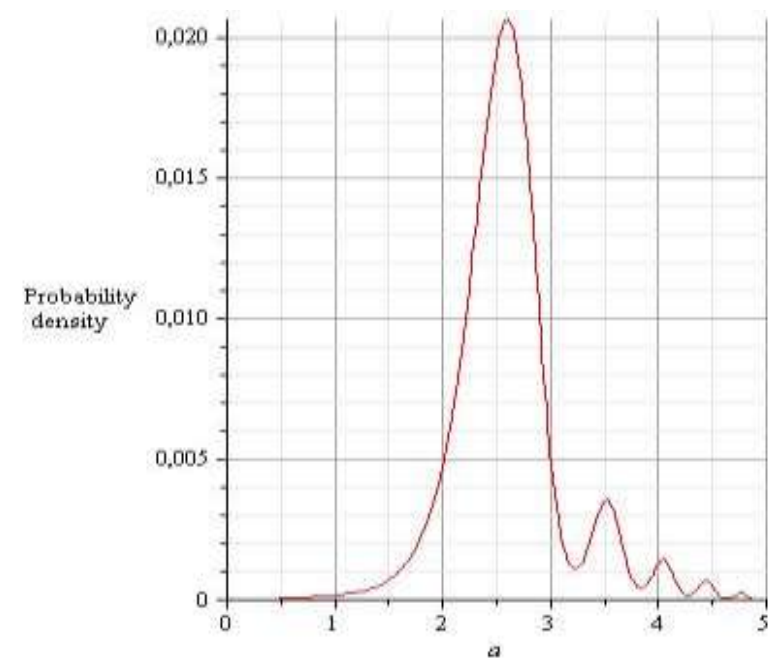

Figura 4: Densidade de probabilidade inicial $|\psi(a, 0)|^{<}$(vácuo). 
Finalmente, para o caso de paredes de domínio, o potencial efetivo quântico é dado por

$$
\begin{gathered}
Q_{e f}(a)=144 k a^{2}-24 p_{\text {Tradiação }}-24 p_{\text {Tpoeira }} a- \\
24 p_{\text {Tcordas }} a^{2}-24 p_{\text {Tvácuo }} a^{4} .
\end{gathered}
$$

Para este caso o valor esperado do fator de escala do Universo e a densidade de probabilidade inicial assume seguinte comportamento:

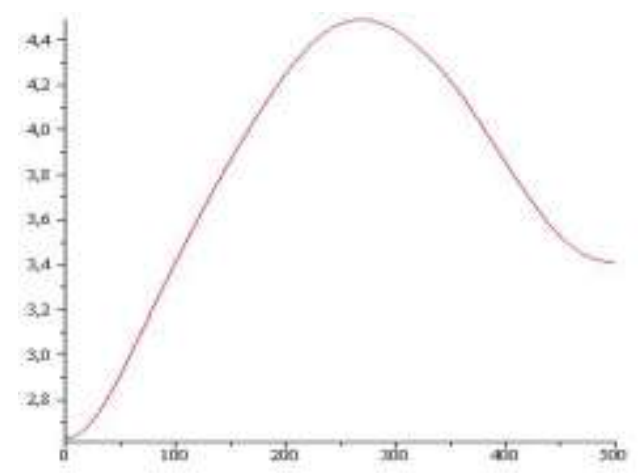

Figura 5: Valor esperado <a>, k=1, em função do tempo $t$ (paredes).

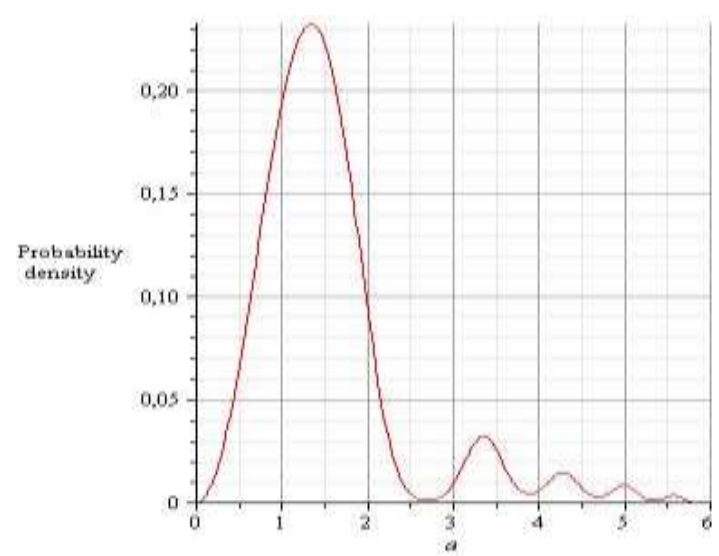

Figura 6: Densidade de probabilidade inicial $|\psi(a, 0)|^{<}$(paredes).

Para todos os casos analisados relativos aos diferentes fluidos, os pacotes de onda foram construídos a partir da superposição dos dez primeiros autoestados mais baixos.

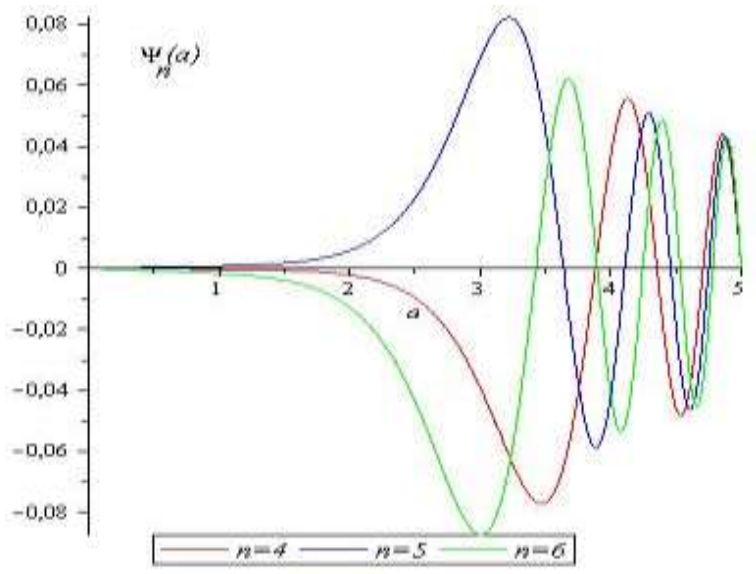

Figura 7: Exemplos de autoestados para o caso vácuo.
O espectro de energia para os diferentes fluidos, pode ser comparado via a tabela abaixo, em função do parâmetro a da equação de estado característica, $p=\alpha \rho$ :

\begin{tabular}{|l|l|l|l|}
\hline $\mathrm{n}$ & $\alpha=-1$ & $\alpha=-1 / 3$ & $\alpha=-2 / 3$ \\
\hline 1 & 0.00320762158 & 0.0638443492231 & 5.8223970065 \\
& 3758755 & 5227 & 43908 \\
\hline 2 & 0.00532690984 & 0.07128227629787 & 5.83886631722 \\
& 7715984 & 075 & 2031 \\
\hline 3 & 0.00763539004 & 0.07902844926431 & 5.85380398174 \\
& 0493943 & 699 & 324 \\
\hline 4 & 0.01021564075 & 0.08744876364554 & 5.86818815967 \\
& 340668 & 699 & 7519 \\
\hline 5 & 0.01310752731 & 0.09672396083012 & 5.88223818866 \\
& 126028 & 484 & 7222 \\
\hline 6 & 0.01633789384 & 0.10698006638195 & 5.89588998044 \\
& 589971 & 09 & 298 \\
\hline 7 & 0.01992726387 & 0.11832490438535 & 5.91017825659 \\
& 326683 & 23 & 9919 \\
\hline 8 & 0.02824635268 & 0.13087047909198 & 5.92713246382 \\
& 596605 & 83 & 4033 \\
\hline 9 & 0.02824635268 & 0.14474065002100 & 5.94726422824 \\
& 596605 & 58 & 6806 \\
\hline 10 & 0.03300138081 & 0.16005711308435 & 5.97032562356 \\
& 636231 & 93 & 8643 \\
\hline
\end{tabular}

Tabela 1: Autoenergias calculadas pelo método espectral de Galerkin.

Observa-se que os valores de $E$ crescem com a diminuição de a para os casos analisados.

Todas as soluções de valor esperado do fator de escala evidenciam Universos que nascem não singulares. Entretanto, para o cenário de vácuo não é observada uma fase de contração, evidenciando a característica de expansão do cenário tipo Big Bang.

Os potenciais efetivos para todos os casos também podem ser comparados. Para o caso de fluido de cordas, tem-se:

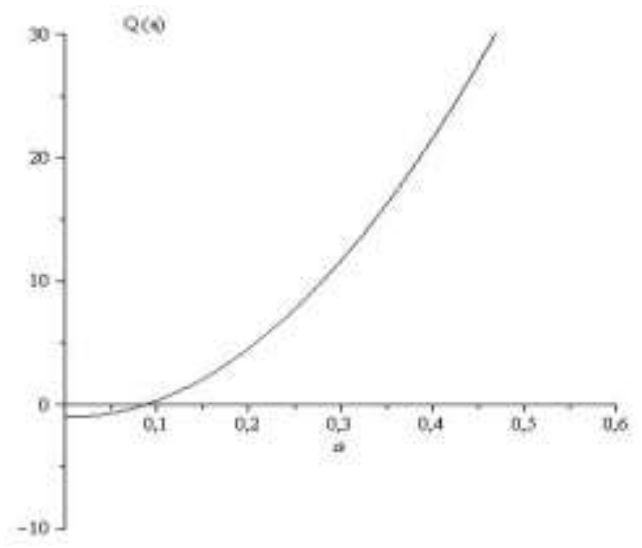

Figura 8: Potencial efetivos, $\mathrm{k}=1$ (cordas).

No caso de vácuo, o potencial assume a forma de uma barreira de potencial, sugerindo a possibilidade do processo de tunelamento quântico da região classicamente proibida para a permitida, conforme previsto em [6]: 


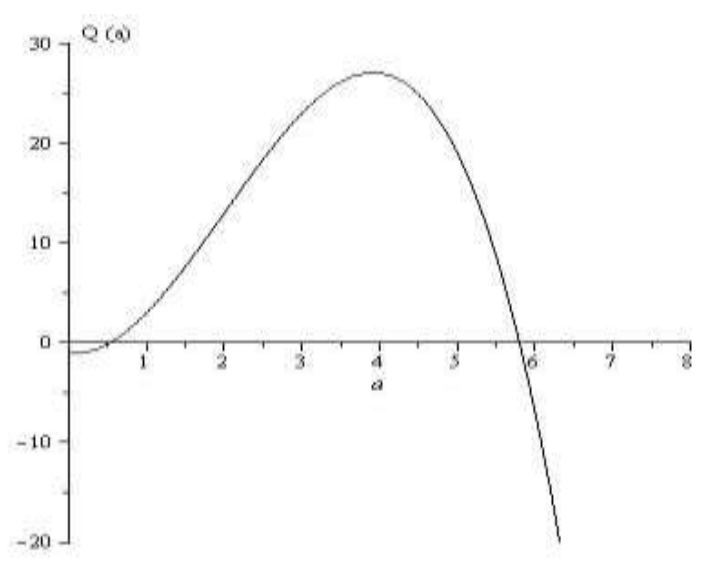

Figura 9: Potencial efetivo, k=1 (vácuo).

O comportamento do potencial para o caso de fluido de paredes de domínio é semelhante ao de cordas:

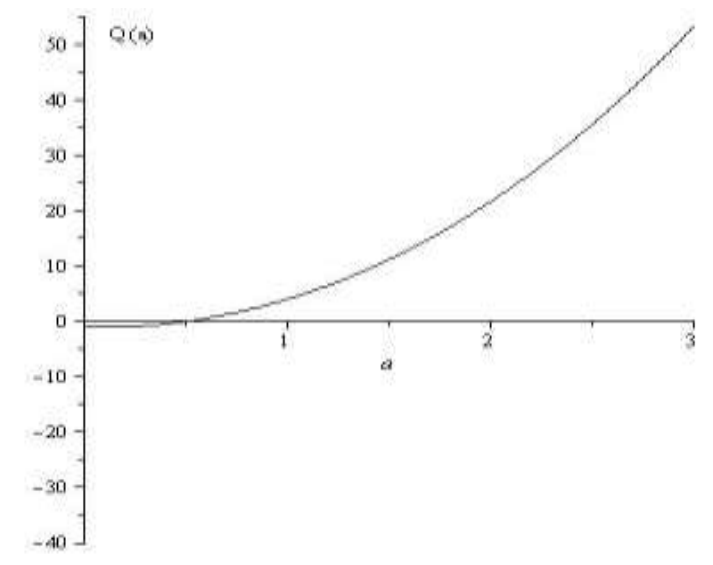

Figura 10: Potencial efetivo, $k=1$ (paredes).

\section{Conclusões e Perspectivas}

A possibilidade de emprego da matéria para exercer o papel do tempo em modelos cosmológicos quânticos gera o questionamento de qual conteúdo material seria o mais adequado. Em um modelo com cinco fluidos, ao comparar os resultados de valores esperados para o fator de escala do Universo para os casos de cordas cósmicas, vácuo e paredes de domínio, observa-se uma maior possibilidade de evolução para o cenário do Universo Big Bang no caso de vácuo, onde está bem caracterizada a fase de expansão do Universo e a possibilidade de nascimento do mesmo via um processo de tunelamento quântico.Todos os casos foram analisados com curvatura espacial k=1.

É importante ressaltar que este é um toy model que objetiva estudar o comportamento matemático das diferentes soluções cosmológicas obtidas. O emprego das constantes dimensionais possibilitará em um trabalho futuro testar os diferentes casos estudados com dados observacionais e então prever qual melhor modelo evoluirá, por exemplo, para a fase inflacionária.

Os casos relativos aos demais fluidos (poeira e radiação) como candidatos ao tempo, estão em fase de análise preliminar, e serão discutidos também posteriormente.

\section{Agradecimentos}

Os autores agradecem à FAPES pelo apoio financeiro.

\section{Referências}

[1] HALLIWELL, J. A. Quantum Cosmology and Baby Universes, ed. S. Coleman, J. B. Hartle, T. Piran and S Weinberg (World Scientific, Singapore, 1991).

[2] ANDERSON, E. The Problem of Time in Quantum Gravity (capítulo 4 de Classical and Quantum Gravity: Theory, Analysis and Applications), ed. V. R Frignanni (Nova, New York 2012).

[3] AlVARENGA, F. G.; FURTADO, R. G.; e GONÇALVES, S. V. B. Brazilian Journal of Physics, v. 47, p. 96 (2016).

[4] ALVARENGA, F. G.; FABRIS, J.C.; LEMOS, N.A; e MONERAT, G. A. Gen. Rel. Grav. 34, 65 (2002).

[5] SILVA, E.V.C., MONERAT, G. A., OLIVEIRA NETO, G. de, e FERREIRA FILHO, L.G. Computer Physics Communications, 185, 380 (2014).

[6] ATKATZ, D., American Journal Physicas, 62 (7), (1994). 